\title{
Automatic picking using coherence measurement and global optimization
}

\author{
Marcelo Jorge Luz Mesquita ${ }^{1}$, João Carlos Ribeiro Cruz ${ }^{1}$, Raphael Di Carlo Silva dos Santos ${ }^{12}$ \\ 1 Universidade Federal do Pará \\ ¿Universidade Federal do Oeste do Pará
}

Copyright 2019, SBGf - Sociedade Brasileira de Geofísica

This paper was prepared for presentation during the $16^{\text {th }}$ International Congress of the Brazilian Geophysical Society held in Rio de Janeiro, Brazil, 19-22 August 2019.

Contents of this paper were reviewed by the Technical Committee of the $16^{\text {th }}$ International Congress of the Brazilian Geophysical Society and do not necessarily represent any position of the SBGf, its officers or members. Electronic reproduction or storage of any part of this paper for commercial purposes without the written consent of the Brazilian Geophysical Society is prohibited.

\section{ABSTRACT}

Time picking is an essential step in seismic processing and seismic inversion. We introduce a new automatic scheme to pick and estimate traveltimes of events using the global optimization algorithm Very Fast Simulated Annealing and coherence measurement from the semblance function. The preliminary results in synthetic and real data case showed good convergence for choosing the time samples with better followed the events.

\section{INTRODUCTION}

In seismic processing, time picking is an important step to perform static corrections, time-depth conversion and tomography techniques (Landa et al., 1989; Rezende et al., 2015, Mesquita et al., 2019). This process is usually performed manually and it may contain misfits associated with the subjectivity of the interpreter. To overcome these errors, an automatic and reliable method of the first-arrivals time picking became necessary. Hatherly (1982) introduced statistical-based methods to pick first arrivals and Song et al. (2013) used a multi-time energy ratio windowing to pick primary events. Latif and Mousa (2016) used the radon transform to perform an automatic picking increasing the Copper's method. Furthermore, energybased methods became frequently used (Spagnolini, 1991; Coppens, 1985; Murat and Rudman, 1992) together with fraction dimension analysis and wavelet transform (Boschetii, 1996; Tibuleac et al., 2003).

The automatic picking process can also be considered an optimization problem and a parameter search. Very Fast Simulated Annealing (VFSA) (Ingber, 1989) is a global multiparameter-search algorithm, which uses a statistical guidance to reach a global minimum. Furthermore, it has a wide application to solve problems in geophysics, e.g. Common-Reflection-Surface (CRS) stacking, velocity inversion and compressional-shear waves separation (Garabito et al., 2017; Mesquita et al. 2019; Santos, 2018).

In this work, we introduce a new algorithm of first-arrival time picking of stacked and migrated sections based on VFSA and coherence measurement. The preliminary results showed a good convergence of the method in order to find the points that best followed the seismic events and estimated the first-arrivals time.

\section{THEORETICAL ASPECTS}

\section{Global Optimization (VFSA)}

We implement the method by using the VFSA optimization algorithm, which is a modification of simulated annealing (SA) (Kirkpatrick et al., 1983) algorithm proposed by Ingber (1989). The optimization process involves simulating the evolution of the physical system as it cools and anneals into a state of minimum energy (Sen and Stoffa, 2013).

Ingber (1989) describes the new approach of SA, the socalled VFSA algorithm, as follows: Consider a model parameter $m_{i}^{k}$ in dimension $i$ generated at annealing step $k$ with the range

$$
m_{i}^{k} \in\left[m_{i}^{\min }, m_{i}^{\max }\right],
$$

where $m_{i}^{\min }$ and $m_{i}^{\max }$ are the minimum and maximum values of $m_{i}$. This model parameter is calculated with the random variable $y_{i} \in[-1,1]$ at iteration $k+1$, after a perturbation, using the following relation

such that

$$
m_{i}^{k+1}=m_{i}^{k}+y_{i}\left(m_{i}^{\max }-m_{i}^{\min }\right),
$$

$$
m_{i}^{k+1} \in\left[m_{i}^{\min }, m_{i}^{\max }\right]
$$

Given a uniform distribution $U[0,1]$ and a random number $u_{i} \in U[0,1], y_{i}$ is generated through the expression

$$
y_{i}=\operatorname{sgn}\left(u_{i}-\frac{1}{2}\right) T_{i}\left[\left(1+\frac{1}{T_{i}}\right)^{\left|2 u_{i}-1\right|}-1\right],
$$

such that the global minimum can be statistically obtained by using the cooling schedule

$$
T_{i}(k)=T_{0 i} \exp \left(-c_{i} k^{\frac{1}{D}}\right),
$$

where $T_{0 i}$ indicates the initial temperature for model parameter $i, c_{i}$ is the parameter that tunes the cooler rate and $D$ represents the dimension of the problem.

The process of optimization follows the Metropolis criterion (Metropolis et al., 1953): given the energy perturbation

$$
\Delta E=E\left(\mathbf{m}^{k+1}\right)-E\left(\mathbf{m}^{k}\right),
$$

if $\Delta E \leq 0$, the new model is always accepted. However, if $\Delta E>0$, then the new model is accepted with probability $P$, defined as 


$$
P=\exp \left(-\frac{\Delta E}{T}\right) .
$$

So, generate a random number $r \in U[0,1]$. If $P>r$, then the new model is accepted, otherwise, is rejected. Random moves at each $T$ are performed accepting and rejecting models according to the adopted criterion, so that the probability of achieving the best model increases. Then, in the new iteration, the temperature is lowered repeating the process. Defining $T_{0}$ and $c$, in practical applications, is a problem-dependent.

\section{Coherence Measurement (Semblance)}

An important point in our strategy is the coherence measurement. The optimum $t$ is obtained by finding the maximum coherency calculated for a set of traces in a time window. So, let us adopt the semblance function (Neidell and Taner, 1971) to estimate the presence or absence of signals correlated. The semblance function $S$, that varies between 0 and 1 , is given by

$$
S=\frac{\sum_{t}\left[\sum_{i=1}^{M} A_{i, t(i)}\right]^{2}}{M \sum_{t}\left[\sum_{i=1}^{M} A_{i, t(i)}^{2}\right]},
$$

where $A_{i, t(i)}$ is the seismic signal amplitude indexed by the trace order number and the traveltime $t(i), \Sigma_{t}$ is the time window for coherency measure and $M$ is the number of traces in a set of traces (stacked or migrated seismic section).

\section{Determination of traveltime picking}

The main objective of this work is the determination of traveltimes of events in the neighborhood of a given point in stacked or migrated seismic sections. The process starts with the choice of a first picking point $P_{1}$ (Figure 1), considering a previous interpretation of the event in the seismic section.

The next step is to choose a set of seismic traces from the previously selected trace containing the first picking point $P_{1}$ and a point to be determined. In Figure 1, for example, this set is composed of ten seismic traces. Afterward, we determine a search range that must contain the searched for point of the seismic events. For this, we use as reference the last seismic trace of the definite set. This can be done automatically as a function of the previously estimated point, considering the same as the center point of this new interval and the initial picking for the next search.

In the following step, we start the automatic search of all the traveltimes in the target event. This search considers the measure of coherence of the set of seismic traces chosen. The process can be performed in whole stacked and migrated sections or in parts, depending on the complexity of the medium. Let us consider in this work that the events in seismic sections are simple and not multiple (e.g. pinchout).

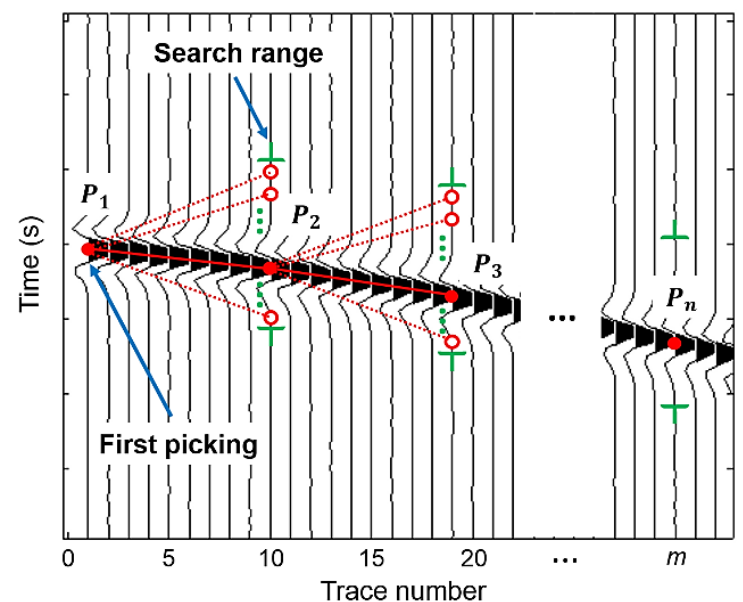

Figure 1. Scheme showing the processing of automatic picking. The green braces represent the search range (maximum and minimum value of $t$ ). The first picking point $P_{1}$ is chosen considering a previous interpretation of the event in the seismic section. From $P_{2}$ to $P_{n}$, all points are estimated automatically.

\section{EXAMPLES}

\section{Synthetic case}

Considers a homogeneous velocity model (Figure 2) composed of six layers with smooth interfaces and constant velocities: $v_{1}=1508(\mathrm{~m} / \mathrm{s}), v_{2}=1581(\mathrm{~m} / \mathrm{s})$, $v_{3}=1690(\mathrm{~m} / \mathrm{s}), v_{4}=1826(\mathrm{~m} / \mathrm{s}), v_{5}=2000(\mathrm{~m} / \mathrm{s})$ and $v_{6}=$ $2236(\mathrm{~m} / \mathrm{s})$.

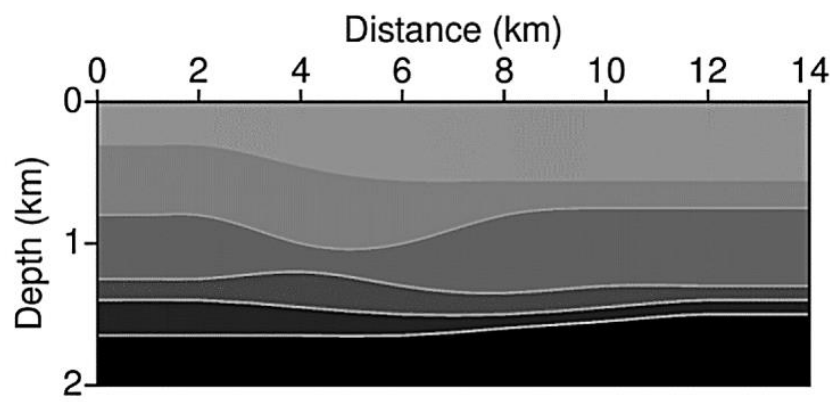

Figure 2. A homogeneous seismic velocity model with six layers and smooth interfaces (Rezende et al., 2015).

After the simulated acquisition and the standard seismic data processing, we obtained a stacked seismic section (Rezende et al., 2015) in which we applied the proposed method. The section in question is composed of five continuous events, corresponding to the interfaces between the layers.

\section{Real case}

For the real case, we applied the proposed method to a part of the time-migrated section from the Tacutu Basin (Garabito et al., 2017), as shown in Figure 3. The choice of the selected region is given by patterns of events with considerable curvatures. 


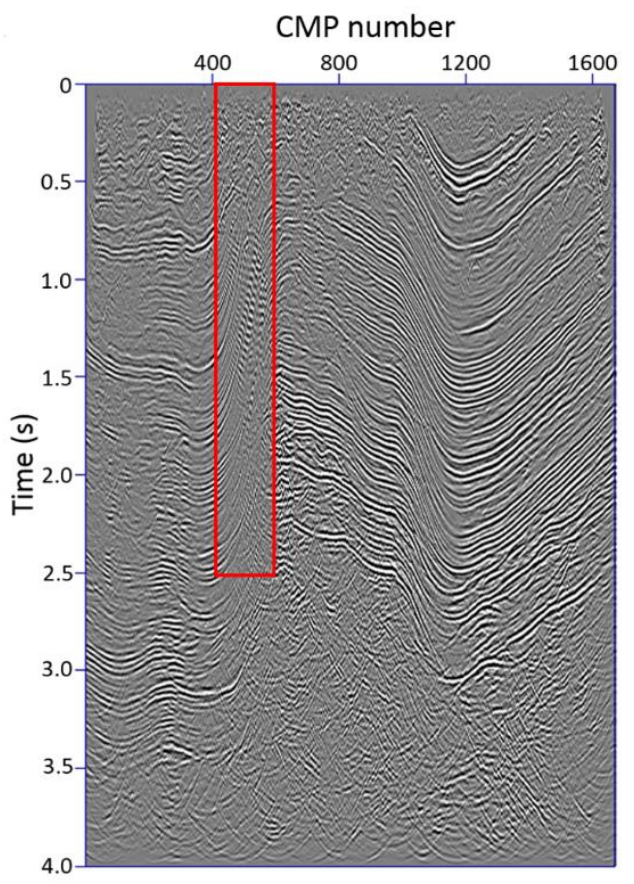

Figure 3. Kirchhoff PSTM of the pre-processed data with enhancement by the FO CRS stacking (modified from Garabito et al., 2017).

\section{RESULTS}

For the synthetic case, we performed the tests in the stacked section. For the optimization process, we choose the VFSA main parameters $T_{0}=0.3$ and $c=0.2$. Table 1 presents, for each event, the number of iterations, the search range (centered in $t_{i}, i=2, \ldots, n$ ) and the time of the first picking $P_{1}$, respectively.

Table 1. Parameters used in optimization process for the synthetic case.

\begin{tabular}{|c|c|c|c|}
\hline & $\mathrm{k}$ & Search range & $t_{1}(\mathrm{~s})$ \\
\hline Event 1 & 500 & {$\left[t_{i}-0.06 ; t_{i}+0.06\right](\mathrm{s})$} & 0.41 \\
\hline Event 2 & 600 & {$\left[t_{i}-0.06 ; t_{i}+0.06\right](\mathrm{s})$} & 1.30 \\
\hline Event 3 & 500 & {$\left[t_{i}-0.04 ; t_{i}+0.04\right](\mathrm{s})$} & 1.57 \\
\hline Event 4 & 500 & {$\left[t_{i}-0.02 ; t_{i}+0.02\right](\mathrm{s})$} & 1.73 \\
\hline Event 5 & 600 & {$\left[t_{i}-0.02 ; t_{i}+0.02\right](\mathrm{s})$} & 1.98 \\
\hline
\end{tabular}

The result is shown in Figure 4, which corresponds to the region between $2 \mathrm{~km}$ and $12 \mathrm{~km}$ of the model. The picking points are represented in red color and show that the method worked very well in this case. The first picking is in the first trace of the section (Figure 4).

During the process, we notice the presence of multiple optimal solutions, this occurs because the random moves of the parameter $t$ can be very small, so that the semblance value does not modify substantially. The solution found was to add the maximum value of semblance to the chosen time whose amplitude is also the maximum. This type of heuristic solution tends to be modified in the course of developing the method.

One of the advantages found in this process is that the value of the slope for each group of traces in the calculation of the semblance gives us the term $d t / d x$ directly by the optimization process for the estimated point. With this information and the velocity near to the surface, we can calculate the emergence angles of image rays that start at the surface and travel to the picking-points (Robein, 2003).

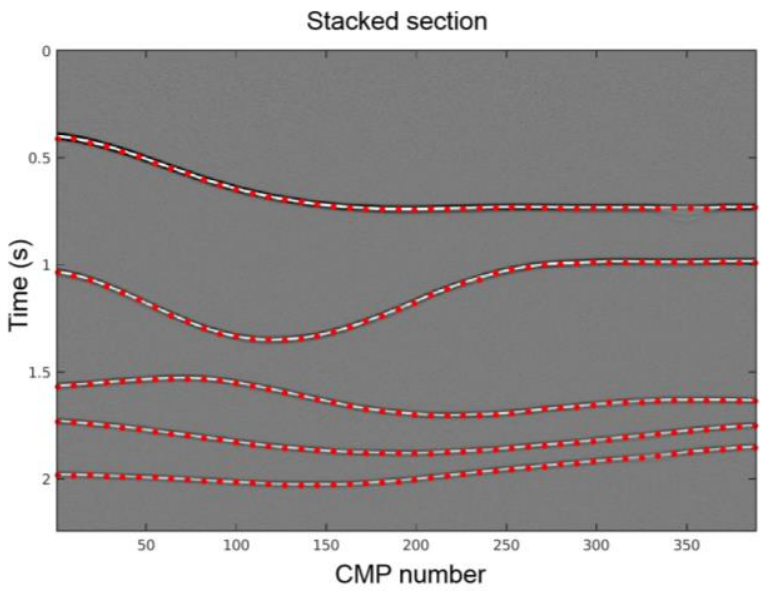

Figure 4. Stacked section and the result of picking (in red color) for each interface.

Figure 5 presents the process of construction of each interface in depth of the studied model with the use of normal rays and the knowledge of the velocity model.

For the real case, we performed the tests in a timemigrated section. For the optimization process, we choose the VFSA main parameters $T_{0}=0.3$ and $c=0.2$. Table 2 follows the same pattern as the synthetic case. Figure 6 shows the result of the test.

The result showed that the picking points follow the chosen events considerably. The addition of a priori information (more than one "first picking") would contribute to a better result in more complex cases.

Table 2. Parameters used in optimization process for the real case.

\begin{tabular}{|c|c|c|c|}
\hline & $\mathrm{k}$ & Search range & $t_{1}(\mathrm{~s})$ \\
\hline Event 1 & 500 & {$\left[t_{i}-0.06 ; t_{i}+0.04\right](\mathrm{s})$} & 0.75 \\
\hline Event 2 & 600 & {$\left[t_{i}-0.06 ; t_{i}+0.04\right](\mathrm{s})$} & 0.99 \\
\hline Event 3 & 500 & {$\left[t_{i}-0.08 ; t_{i}+0.02\right](\mathrm{s})$} & 1.19 \\
\hline Event 4 & 500 & {$\left[t_{i}-0.08 ; t_{i}+0.02\right](\mathrm{s})$} & 1.50 \\
\hline Event 5 & 600 & {$\left[t_{i}-0.08 ; t_{i}+0.02\right](\mathrm{s})$} & 1.67 \\
\hline Event 6 & 500 & {$\left[t_{i}-0.06 ; t_{i}+0.02\right](\mathrm{s})$} & 1.85 \\
\hline Event 7 & 500 & {$\left[t_{i}-0.10 ; t_{i}+0.02\right](\mathrm{s})$} & 2.10 \\
\hline Event 8 & 500 & {$\left[t_{i}-0.06 ; t_{i}+0.02\right](\mathrm{s})$} & 2.34 \\
\hline
\end{tabular}




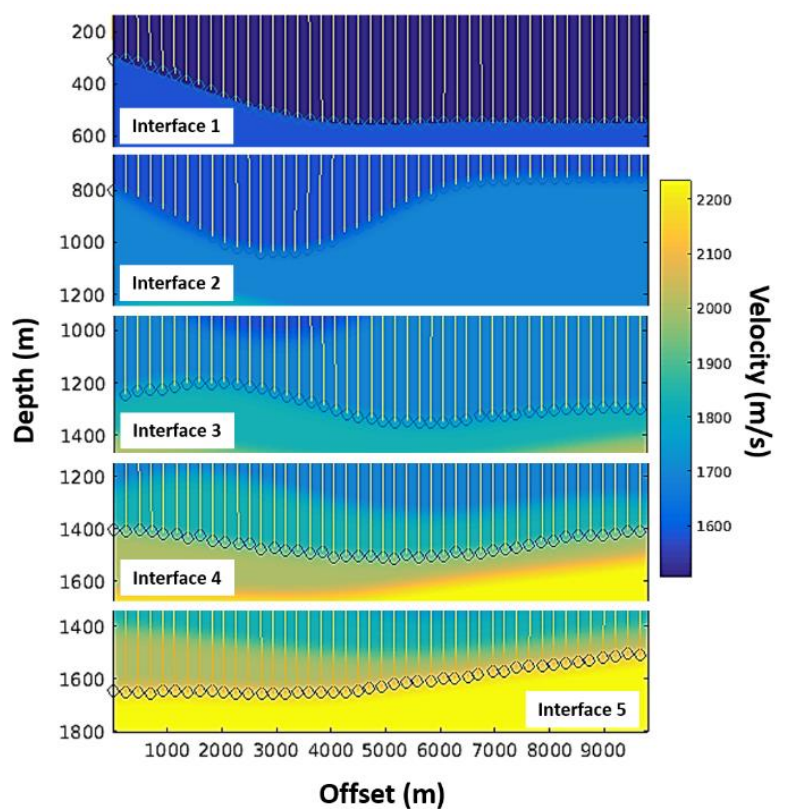

Figure 5. Points in depth of each time-picking points from the stacked section of the synthetic case. This conversion can be done using normal rays and knowing the velocity model.

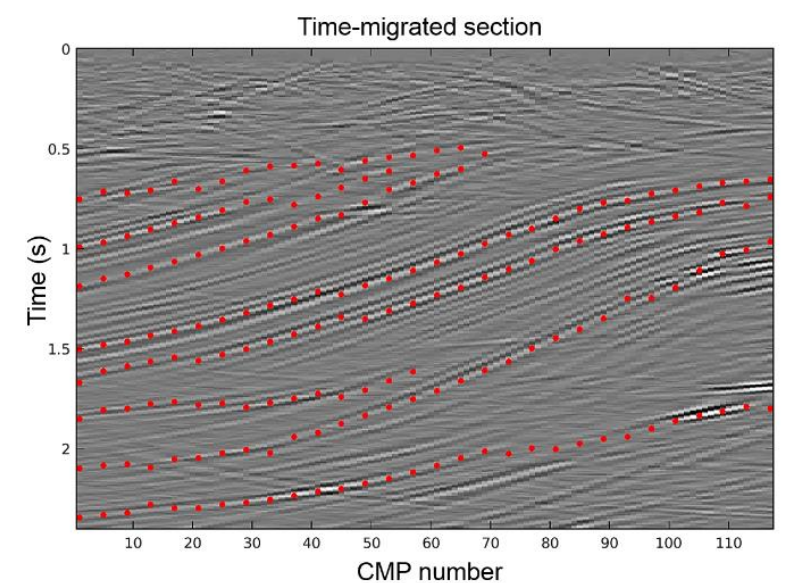

Figure 6. Migrated section and the result of picking for eight selected events.

\section{CONCLUSIONS}

Time picking is an important step in seismic process and inversion. We presented a proposed method of first-arrival time picking of stacked and migrated sections based on VFSA and semblance measurements.

The results show that the algorithm worked very well in both synthetic and real cases, and we may consider the time-picking problem as an optimization problem. In addition, our examples demonstrated that the chosen parametrization collaborated to reaching the desired solution.
This method is still in the refinement phase and some tests, modifications and applications may be needed.

\section{ACKNOWLEDGMENTS}

The authors would like to thank the National Petroleum Agency of Brazil for providing the Tacutu seismic data set and the Laboratory of Seismic Wave Inversion (LSWI) of UFPA.

\section{REFERENCES}

Boschetti, F., 1996, A fractal-based algorithm for detecting first arrivals on seismic traces: Geophysics, 61, 10951102.

Coppens, F., 1985, First arrival picking on common-offset Trace collections for automatic estimation of static corrections: Geophysical Prospecting, 33, 1212-1231

Garabito, G., Cruz, J. C. R., and Söllner, W., 2017, Finiteoffset common reflection surface stack using global optimization for parameters estimation: a land data example: Geophysical Prospecting, 65.

Hatherly, P. J., 1982, A computer method for determining seismic first arrival times: Geophysics, 47, 1431.

Ingber, L., 1989, Very fast simulated reannealing. Mathematical and Computer Modeling, 12(8), 967-993.

Kirkpatrick, S., Gelatt, C. D., and Vecchi, M. P., 1983, Optimization by Simulated Annealing, Science, New Series, V. 220, n. 4598. (May 13, 1983), p. 671-680.

Landa, E., Beydoun, W., and Tarantola, A., 1989, Reference velocity model estimation from prestack waveforms: Coherency optimization by simulated annealing: Geophysics, 54(8), 984-990.

Latif, A., Mousa, W. A., 2016, An efficient and robust method for automatic first arrival picking using undersampled fast high resolution Radon transform. SEG International Exposition and 86th Annual Meeting. 24392443.

Mesquita, M. J. L., Cruz, J. C. R., and Garabito, G., 2019, Velocity inversion by global optimization using finite-offset common-reflection-surface stacking applied to synthetic and Tacutu Basin seismic data, Geophysics, v. 82 (2), p. B121-V132.

Metropolis, N., Rosenbluth, A. W., Rosenbluth, M. N., Teller, A. H., and Teller, E., 1953, Equation of State Calculations by Fast Computing Machines, Journal of Chemical Physics, 21, p. 1087.

Murat, M. E., and Rudman, A. J., 1992, Automated first arrival picking: A neural network approach. Geophysical Prospecting, 587-604

Neidell, N. S., and Taner, M. T., 1971, Semblance and other coherency measures for multichannel data. Geophysics, v. 36, p. 482-497.

Rezende, D. P., Cruz, J. C. R., Fernandes, A. S., Santos, R. C. S., Mesquita, M. J. L., 2015, Time-depth conversion using image ray and normal ray in the tau-p domain: application in synthetic data, $14^{\text {th }}$ International Congress of 
the Brazilian Geophysical Society, Expanded Abstracts, 1095-1099.

Robein, E., Velocities, Time-imaging and Depth-imaging in Reflection Seismics. Principles and Methods. EAGE Publications 2003. Houten, Netherlands. 464 p.

Santos, R.D.C.S. Separation of PP and PS converted waves using finite offset common reflection surface stack. 2018. Universidade Federal do Pará. Phd Thesis. 112 p.

Sen, M. K., and Stoffa, P. L., 2013, Global Optimization Methods in Geophysical Inversion, Cambridge.

Song, J., Cao, X., Xu, W., Yang, J., 2013, First arrival time auto-picking method based on multi-time windows energy ratio. Energy Science and Technology, 6, 79-89.

Spagnolini, U., 1991, Adaptive picking of refracted first arrivals. Geophysical Prospecting, 39, 293-312

Tibuleac, I. M., Herrin, E. T., Britton, J. M., Shumway, R., and Rosca, A. C., 2003, Automatic determination of secondary seismic phase arrival times using wavelet transforms. Seismological Research Letters, 74, 884-892 